\title{
A Rare Cause of Cardiac Tamponade: Left Ventricular Pacemaker Malposition
}

\author{
Srikanth Seethala*,, Anit Kumar ${ }^{\mathrm{b}}$, Gur Charan Adhar $^{\mathrm{c}}$ and Thomas Generalovich ${ }^{\mathrm{c}}$ \\ ${ }^{a}$ Department of Internal Medicine, Texas Tech University Health Sciences Center, El Paso, USA; ${ }^{b}$ University of Pitts- \\ burgh-Mercy Hospital, Pittsburgh, USA; ${ }^{c}$ Midtown Cardiovascular Associates, Pittsburgh, Texas Tech University \\ Health Sciences Center, USA
}

\begin{abstract}
The malpositioning of a pacemaker lead is an uncommon and under-reported complication of pacemaker/Automated Implantable Cardioverter-Defibrillator (AICD) placement. It can be associated with complications such as pericardial effusions and tamponade. We describe a case of an elderly woman who developed cardiac tamponade after inadvertent placement of a permanent pacing lead in the left ventricle. Following implantation of the permanent pacemaker, the patient developed dyspnea, and hypotension. Echocardiogram showed evidence of cardiac tamponade. Patient was successfully treated with pericardiocentesis and later on repositioning of the pacemaker lead in right ventricle.
\end{abstract}

Keywords: Malposition of the pacemaker lead in left ventricle, cardiac tamponade, perforation.

\section{INTRODUCTION:}

Inadvertent placement of pacemaker/AICD lead in the left ventricle is a rare and under diagnosed complication. It can be associated with the complications like myocardial perforation and pericardial effusions.

\section{CASE}

An 81 year-old female with a history of diabetes, cerebrovascular accident, hypertension, and no known structural heart disease was admitted with recurrent syncope to a regional health center. She was observed to have brief sinus pauses on holter monitor. On telemetry, she had a prolonged asystolic sinus pause of 12 seconds with syncope. The patient was taken to the operating room for dual-chamber permanent pacemaker placement. During the procedure, the patient was observed to be in bradycardia with a sinus rhythm at a heart rate of 30 beats per minute. A note from the implanting physician indicated difficulty in positioning the ventricular lead. Within three hours of pacemaker implantation, the patient reported acute shortness of breath and became hypotensive. Echocardiogram showed moderate pericardial effusions and cardiac tamponade. The patient was transferred to our institution for possible lead perforation. After confirming cardiac tamponade with portable echo, 170 -cc of bloody pericardial fluid was aspirated resulting in immediate improvement of blood pressure and symptoms. A pericardial drain was left in place for ventricular perforation as a possible cause of cardiac tamponade. An EKG revealed atrial sensing and a ventricular paced rhythm with the morphology of a right bundle branch block (RBBB) and right axis deviation (Fig. A). A repeat post-pericardiocentesis echocardiogram revealed the ventricular lead at the left

\footnotetext{
*Address correspondence to this author at the Dept of Internal Medicine, 4800 Alberta Ave, El Paso, TX 79905, USA; Tel: (972) - 835-2873; Fax: 915- 545-6699; E-mail: srikanth.seethala@yahoo.com;

srikanth.seethala@ttuhsc.edu
}

ventricular apex passing through the interatrial septum in the region of the fossa ovalis (Fig. B, C). The chest X-ray revealed an abnormal ventricular lead location (Fig. D). On the following date, the lead was repositioned in the right ventricle. The pericardial drain was removed and the patient remained stable and was discharged home after two days.

\section{DISCUSSION}

Inadvertent placement of pacemaker/AICD lead in the left ventricle is a rare and under diagnosed complication [1]. Malposition of the lead in the left ventricle may be secondary to inter atrial-septal defect, left subclavian artery cannulation, inter-ventricular septal defect, interventricular septal perforation, or due to placement of lead through the coronary sinus into its tributaries [1]. Rarely, inadvertent left ventricular epicardial stimulation can also occur as a result of lead migration to the pericardial surface through a perforated right ventricular apex [2]. Inadvertent left ventricular lead placement is associated with complications such as systemic thromboembolism, myocardial perforation, and damage to cardiac structures such as the mitral and aortic valves $[3,4]$. The longest unrecognized case of inadvertent left ventricular lead placement has been recorded as 17 years [5].

The diagnosis of the left ventricular lead (LVL) malposition requires a high index of suspicion. A RBBB pattern in the anterior precordial leads (V1, V2) occasionally can be seen with pacing from the right ventricle. However, in such a scenario, standard limb leads show a LBBB pattern and the final QRS vector is oriented to the left, superior, and anterior direction (pseudo RBBB). In contrast to pseudo RBBB, the final vector of left ventricular pacing is oriented to the right, inferior and posterior. Pseudo RBBB can be eliminated by recording the V1 and V2 leads one intercostal space lower than the normal [6]. Chest roentgenography can be helpful in identifying the lead position, and 2D-echocardigram is useful in confirming the diagnosis [3]. 


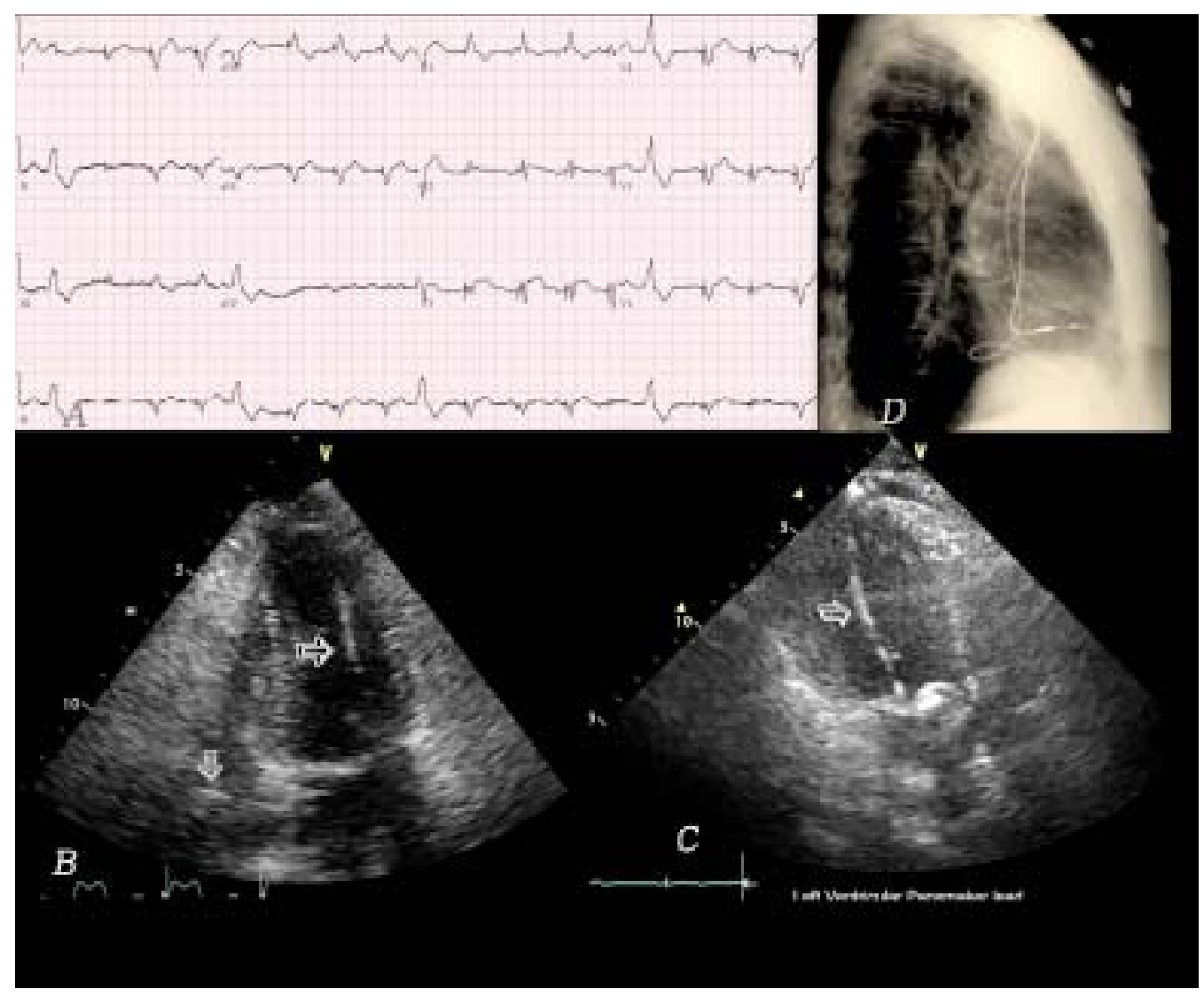

Fig. (1). A. EKG showing atrial sensing and ventricular paced rhythm with the morphology of a right bundle branch block (RBBB) and right axis deviation; B: Apical 2 chamber view showing pacemaker lead in left ventricle and small apical pericardial effusion. C: Apical 4 chamber view showing pacing lead passing through interatrial septum, mitral valve. D: Lateral chest X-ray revealing an abnormal loop of the ventricular lead.

Our Special thanks to Ms. Valerie Shive, for support.

There is no consensus on the management of LVL malposition. The reported incidence of systemic thromboembolic complications is as high as $37 \%$ [4]. Therapeutic options include leaving the lead in place and reducing the risk of thromboembolic disease by using long term oral anticoagulation agents, or removal/repositioning of the lead [3, 4]. If the LVL is recognized soon after implant, repositioning of the lead in the right ventricle is the desired treatment [3].

Myocardial perforation during permanent pacer/AICD placement is an uncommon but well-recognized complication. Prevalence of myocardial perforation during pacemaker placement varies between $0.1-0.8 \%$ and that of AICD placement is between 0.6-5.2\% [7]. Risk factors for myocardial perforation include lead design, operator experience, old age (age > 85), steroid use, low body mass index, and temporary pacing [8]. Right atrial perforation is more common than right ventricular perforation [9]. Myocardial perforation clinically may present as an incidental finding on chest Xray or CT scan, loss of sensing or pacing, pericarditis or as an acute/subacute pericardial effusion with or without cardiac tamponade [10]. To our knowledge, there is only a single case report involving left ventricular perforation during single chamber pacemaker placement [10]. There are rare case reports of late pericardial effusion after inadvertent LVL placement [1]. The site of perforation in our case is unclear. Even though we suspected thick left ventricular wall perforation based on difficulty in placing the ventricular lead, the most likely cause of tamponade was right atrial perforation, since this is the most common location [9].

\section{REFERENCE LIST}

[1] Engstrom A, Holmberg B, Mansson A, Carlsson J. Inadvertent malposition of a transvenous pacing lead in the left ventricle. Herzschrittmacherther Elektrophysiol 2006; 17: 221-4.

[2] Van Gelder BM, Brackle FA, Oto A, et al. Diagnosis and management of inadvertently placed pacing and ICD leads in the left ventricle: a multicenter experience and review of the literature. Pacing Clin Electrophysiol 2000; 23: 877-83.

[3] McManus DD, Mattei ML, Rose K, Rashkin J, Rosenthal LS. Inadvertent lead placement in the left ventricle: a case report and brief review. Indian Pacing Electrophysiol J 2009; 9: 224-8.

[4] Seki H, Fukui T, Shimokawa T, et al. Malpositioning of a pacemaker lead to the left ventricle accompanied by posterior mitral leaflet injury. Interact cardiovasc thorac surg 2009; 8: 235-7.

[5] Van Erckelens F, Sigmund M, Lambertz H, Kreis A, Reupcke C, Hanrath P. Asymptomatic left ventricular malposition of a transvenous pacemaker lead through a sinus venosus defect: follow-up over 17 years. Pacing Clin Electrophysiol 1991; 14: 989-93.

[6] Yang YN, Yin WH, Young MS. Safe right bundle branch block pattern during permanent right ventricular pacing. J Electrocardiol 2003; 36: 67-71.

[7] Khan MN, Joseph G, Khaykin Y, Ziada K, Wilkoff B. Delayed lead perforation: A disturbing trend. Pacing Clin Electrophysiol 2005; 28: 251-3.

[8] Sterlinski M, Przybylski A, Maciag A, et al. Subacute cardiac perforations associated with active fixation leads. Europace 2009; 11: 206-12. 
[9] Hirschl DA, Jain VR, Spindola-Franco H, Gross JN, Haramati LB. Prevalance and characterization of asymptomatic pacemaker and ICD lead perforation on CT. Pacing Clin Electrophysiol 2007; 30: 28-32.
[10] Sanchis DV, Laguna MJH, Cordon LPBD. Late left ventricular perforation as a complication of permanent pacing leads. Eur J Intern Med 2006; 17: 72.

(C) Seethala et al.; Licensee Bentham Open.

This is an open access article licensed under the terms of the Creative Commons Attribution Non-Commercial License (http: //creativecommons.org/licenses/by$\mathrm{nc} / 3.0 /$ ) which permits unrestricted, non-commercial use, distribution and reproduction in any medium, provided the work is properly cited. 